\title{
Implications of African genetic diversity
}

A $s$ the birthplace of modern humans, the African continent has been inhabited continuously for $\sim 200,000$ years, at least twice as long as any other continent. Consequently, high levels of genetic diversity exist among different African ethnic groups, and nearly one-third of all languages spoken in the world today are associated with the more than 2,000 ethnolinguistic groups identified in Africa. A large collaborative international research effort (Tishkoff, S. A. et al. Science 324, 1035-1044 [2009]) has given a quantitative account of this genetic diversity that has important implications for the treatment of people of African descent with renal conditions.

The researchers found that all but one of the 121 African ethnic groups studied have more withingroup genetic diversity than any of the 60 non-African ethnic groups for which comparable data were collected. The average number per genetic locus of private alleles-alleles present in only one of many populations sampled-is far higher in Africa than in any other continent. By and large, the pattern of genetic differences observed among African populations matched linguistic and cultural differences. The study also confirmed historical evidence on the slave trade that African Americans can trace most of their ancestry to speakers of the Niger-Kordofanian language family originating from a vast swath of west Africa that spans regions as far west as Senegambia and as far south as Angola and South Africa.

Publication of this study questions whether, and to what extent, catch-all terms like 'African American', 'African ancestry' and 'black' are useful or indeed misleading in medical research and clinical practice. Implications for the nephrology community are farreaching, not least in developed countries with a growing presence of African immigrants. Take for example the case of the aggressive renal condition HIV-associated nephropathy (HIVAN). For reasons that are probably mostly rooted in genetics, about $50 \%$ of all HIV-positive African Americans with kidney disease have HIVAN, whereas this condition is very rare among European
Americans. In studies of black South Africans, the prevalence of HIVAN varies between $10 \%$ and $83 \%$ (Faiban, J. \& Naicker, S. Nat. Rev. Nephrol. 5, 591-598 [2009]), possibly reflecting the high degree of genetic variability among the many aboriginal South African ethnic groups. A study of $126 \mathrm{HIV}$-positive Ethiopians living in Israel failed to identify a single case of HIVAN (Behar, D. M. et al. Am. J. Kidney Dis. 47, 88-94 [2006]). In light of the genetic and clinical evidence, placing a black HIV-positive US resident who has a non-NigerKordofanian ethnic background-such as, for instance, an immigrant or a descendant of immigrants from the horn of Africa-in the same ethnic category as descendents of American slaves of west African ancestry might be scientifically and clinically misleading.

Individuals with different African ethnic backgrounds might have substantially different genetic susceptibility to specific diseases. If this idea is to change clinical practice, however, resolving the issue of genetic versus environmental influences in conditions for which the prevalence is heavily dependent on ethnicity acquires new urgency. A case in point is hypertension, which is more prevalent among African Americans than European Americans. Whether perception of being racially discriminated against - a perception that would probably not differentiate between black individuals of different African ancestry-is a contributing factor in the hypertension ethnicity gap has not been conclusively resolved. This issue is currently so difficult to explore that both study design and data analysis have had to try and take into account psychological coping mechanisms such as anger repression and anger inhibition.

The Science paper suggests that soon it may be possible to unravel the role of human genetic diversity in renal disease. Indeed, new genetic data from people of African ancestry could be an invaluable tool in medical research, one that could help improve our understanding of mechanisms of disease and, crucially, the health care of people from these ethnic groups.

doi:10.1038/nrneph.2009.202

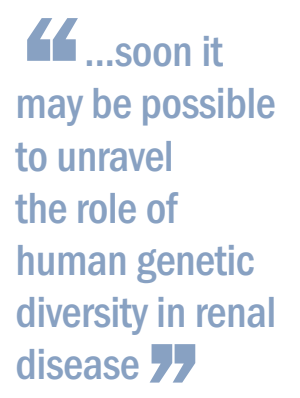

Baldo Lucchese is the Associate Editor of Nature Reviews Nephrology.

Competing interests The author declares no competing interests. 